\title{
Tracking and Understanding Nanocatalyst Sintering and Regeneration using Deep Learning-assisted In Situ Environmental TEM
}

\author{
Rajat Sainju ${ }^{1}$, Steven Suib ${ }^{1}$, Caiwen Ding ${ }^{1}$ and Yuanyuan $\mathrm{Zhu}^{2}$ \\ ${ }^{1}$ University of Connecticut, United States, ${ }^{2}$ University of Connecticut, Storrs, Connecticut, United States \\ *Corresponding author: yuanyuan.2.zhu@uconn.edu
}

Metal nanoparticles have been widely employed in heterogeneous catalysis due to their high surface-tovolume ratios exposing an abundance of surface active sites. However, the active surface of nanocatalyst is also susceptible to catalyst sintering, which results in an increase in nanoparticle size and loss of active metal-surface area. As a major cause of catalyst deactivation especially for high-temperature catalytic reactions, sintering greatly limits a nanocatalyst's lifetime and leads to a high net final energy consumption. Recently, the application of oxidation and reduction cycles to spent catalysts has been demonstrated promising for nanocatalyst regeneration [1]. Yet, the mechanisms of nanocatalyst regeneration remain elusive as there lacks a detailed description of the dynamic nanoparticle transformation in the course of redox cycles.

In this work, we employed in situ environmental transmission electron microscopy (ETEM) and a deep learning-based algorithm to study the sintering and regeneration behavior of nickel nanoparticles during an oxidation-reduction cycle. To mimic the industrial relevant reaction conditions, a base in situ gas pressure up to the atmospheric pressure of 1 bar is needed. Thus, we adopted the state-of-the-art MEMS (Micro-Electro-Mechanical Systems)-based environmental gas cell (DENSsolutions, Climate G+ system) with a flexible dynamic gas-mixing system capable of fast switching between the oxidation and reaction conditions. Unlike conventional sintering studies that rely mostly on measuring averaged nanoparticle size or the overall surface area, the nature of in situ ETEM offers direct real-time visualization of the nanoparticles' evolution at the nanoscale in response to the different reactive gaseous environments. Figure 1 presents representative in situ ETEM snapshots taken from in situ videos demonstrating the size, morphology, and phase transformation of Ni nanoparticles in the course of an oxidation-reduction cycle. We found that the formation of the hollow polycrystalline oxide shell via the nanoscale Kirkendall effect during the oxidation process played an important role in the subsequent regeneration of new and small metallic nanoparticles during reduction. Comparison between Figure 1a and Figure 1c suggests that whether a nanoparticle was sintered or regenerated depends on the intricate interplays among the nanoparticle size, its surrounding nanoparticle species, and the reaction conditions. To facilitate detailed frame-by-frame analysis and enable high throughput quantification of important sintering metrics like particle size and interparticle distance, built on our previous deep learning work [2], we worked on a new branch of computer vision task called multiple object tracking (MOT). MOT not only performs nanoparticle detection in individual video frames it also associates detections with unique nanoparticle IDs and updates nanoparticles' trajectories/appearance in an in situ ETEM video sequence. Figure 2 presents our MOT framework, which utilizes a single-shot joint detection and embedding method that consists of a convolution neural network-based objected detector and a combined reidentification branch for real-time tracking of nanoparticle evolution. In sum, this combination of in situ ETEM and deep learning-based tracker holds the potential to register and scale-up single-particle level analysis that is critical to the understanding of nanocatalyst regeneration [3]. 


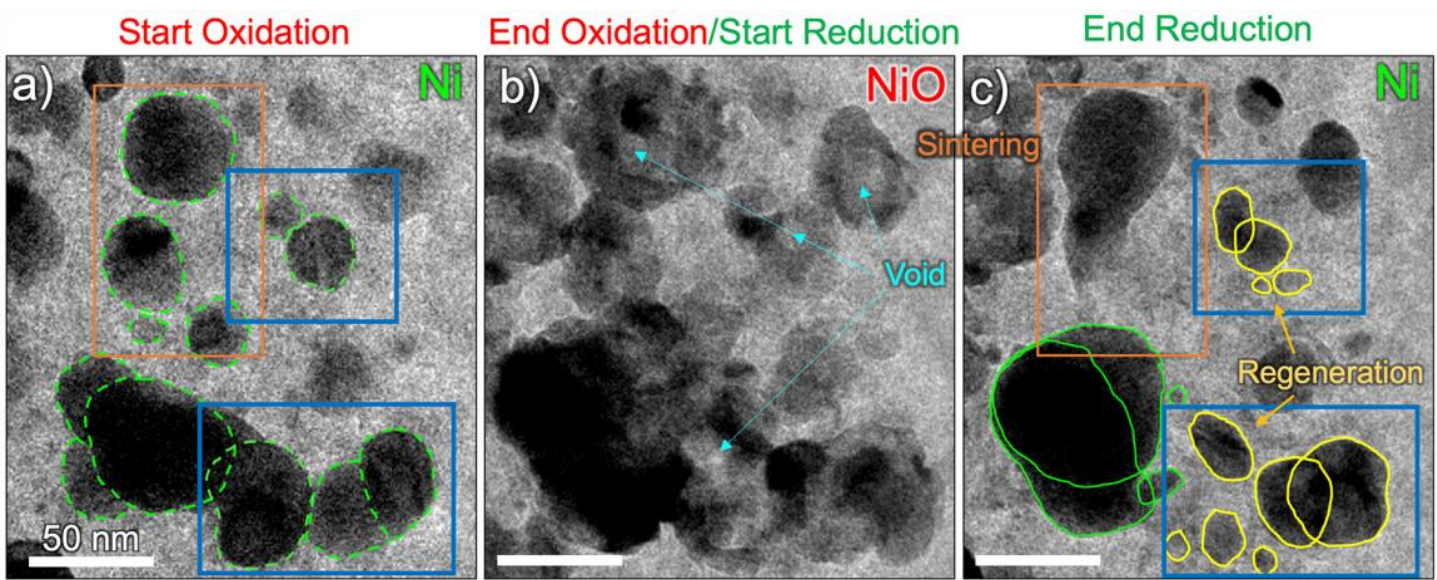

Figure 1. In situ ETEM snapshots of the (a) pristine Ni nanoparticles (outlined by solid green lines), (b) $\mathrm{NiO}$ after oxidation in $\mathrm{O}_{2}$, and (c) new Ni nanoparticles after reduction in $\mathrm{H}_{2}$ at $600{ }^{\circ} \mathrm{C}$. Note that in (c) new small Ni nanoparticles (solid yellow line) were observed regenerated from pristine nanoparticles after the oxidation-reduction cycle. Nanoparticles that sinter is shown in the area marked by the orange rectangle and the nanoparticles that are regenerated by the blue rectangle. Ni nanoparticles that sinters are marked by the orange box and the regenerated ones are marked by the blue boxes.

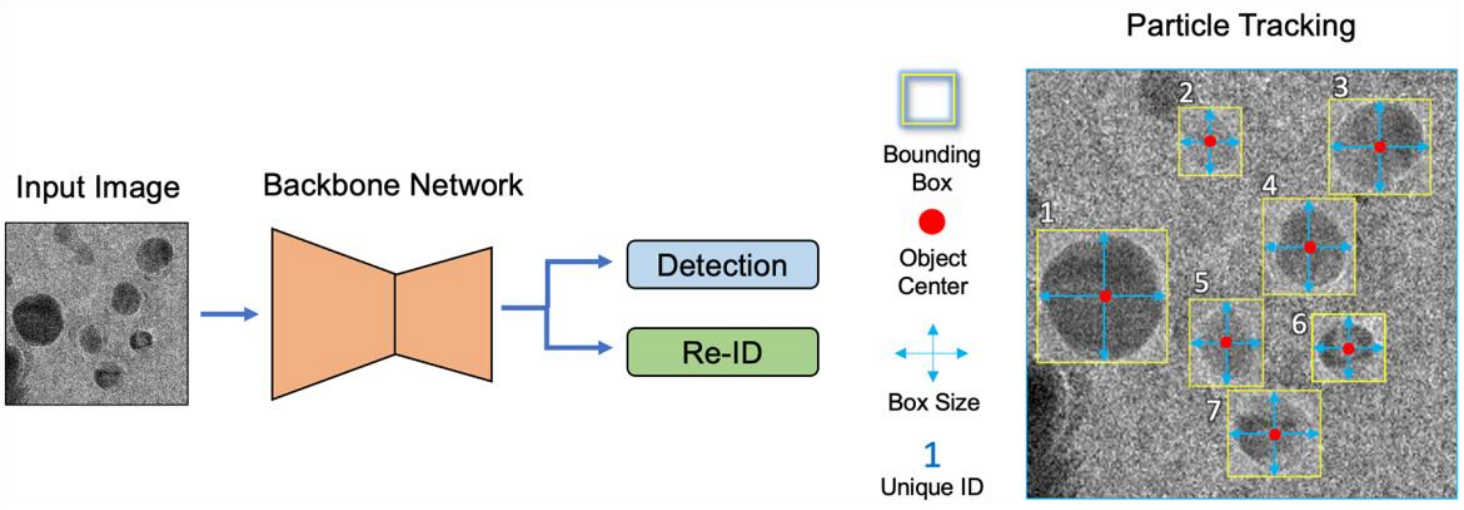

Figure 2. Overview of the single-shot multi-object tracker framework used in this work. The input image is passed through the backbone network for the extraction of high-resolution feature maps, and then the detection and Re-ID branch predict the bounding box and reidentify features for nanoparticle tracking.

\section{References}

[1] DM Argyle and CH Bartholomew, Catalysts, 5 (2015), 145-269.

[2] G Roberts, S Y Haile, R Sainju, D J Edwards, B Hutchinson and Y Zhu, Scientific Reports 9(2019), 12744.

[3] We acknowledge funding from UConn Interdisciplinary Multi-Investigator Materials Proposals (IMMP) award. 